\title{
Beyond Empathy for Pain
}

\author{
Frédérique de Vignemont and Pierre Jacob*广
}

Here we address four objections raised by Julien Deonna, John Michael, and Francesca Fardo against a recent account of empathy for pain (by Frédérique de Vignemont and Tania Singer and Vignemont and Pierre Jacob). First, to what extent must the empathizer share her target's affective state? Second, how can one interpret neuroscientific findings on vicarious pain in light of recent results challenging the notion of a pain matrix? Third, can one offer a simpler account of how empathy makes one aware of another's emotion? Finally, to what extent can this account of empathy for pain be generalized to empathy for emotions?

1. Introduction. It is widely agreed that the primary target of an individual's empathetic response is another's affective state. We have offered a tentative account of empathy for pain based on three further ideas (Vignemont and Singer 2006; Vignemont and Jacob 2012), each of which has recently been subjected to interesting criticisms (Deonna 2007; Michael 2014; Michael and Fardo 2014). First, we assume that empathy and emotional contagion are vicarious experiences. On our minimal definition, an experience is vicarious if (i) it is caused by another person's affective experience and (ii) it is isomorphic (or similar) to some relevant extent to the other's experience. Second, we take vicarious responses to be generated by a process of nonpropositional imagination, whereby one's standard affective resources are used offline, rather than online. Third, we argue that empathetic responses and contagious responses to another's affect have different directions of in-

Received August 2015; revised October 2015.

*To contact the authors, please write to: Frédérique de Vignemont, Institut Jean Nicod, 29 rue d'Ulm, 75005 Paris, France; e-mail: frederique.de.vignemont@ens.fr.

$\dagger$ This work has received support under the program Investissements d'Avenir launched by the French government and implemented by ANR-10-LABX-0087 IEC and ANR10-IDEX-0001-02 PSL. We gratefully acknowledge support of the European Research Council under the European Union's Seventh Framework Program (FP7/2007-2013)/ ERC grant 609819, SOMICS.

Philosophy of Science, 83 (July 2016) pp. 434-445. 0031-8248/2016/8303-0007\$10.00

Copyright 2016 by the Philosophy of Science Association. All rights reserved. 
tentionality: contagious responses are self-centered and empathetic responses are other-directed. Only empathetic experiences meet what we call the ascription condition: they make one aware of the target's affective state. In what follows, we address the major criticisms directed at these basic assumptions. In the final section, we show how this account of empathy for pain can be generalized to empathy for emotions.

2. What Is Shared? One major bone of contention in recent debates about empathy is whether empathy should satisfy the interpersonal similarity condition: to what extent must the empathizer share her target's affective state? Thus, Michael and Fardo $(2014,154)$ ask: "What —if anything — is shared in pain empathy?"

Some philosophers from the phenomenological tradition simply deny that interpersonal similarity is a necessary condition and endorse instead a directperception model of empathy, according to which empathy is what enables one individual to be directly acquainted with another's emotion (Scheler 1954; Zahavi 2011). On this view, one can empathize with another's pain or emotion and not feel anything. If so, then to empathize is simply to know by acquaintance, or to form a perceptual belief about, another's emotion. As a result, as Michael (2014) rightly points out, on this view, there is little difference (if any) between empathizing with another's emotion and mindreading another's emotion. If affective sharing is not required, then how can one draw the required distinction between what is happening to a young child, who cries with Bambi when her mother dies, and the child's father, who is aware of Bambi's distress without sharing it? If we say that both empathize, then we cannot acknowledge the difference any more. Nor could one draw the further distinction between empathy and sympathy, which we propose to construe as a sui generis kind of social affective attitude: no matter what another's affective experience is - pain, jealousy, anger - to sympathize with her is to feel sorry for her. This does not require empathizing with her.

However, one may note that sometimes sympathy seems to meet the interpersonal similarity condition as well. Suppose Y sympathizes with X, who feels sorry because her husband is deeply sick. If Y sympathizes with $\mathrm{X}$, then $\mathrm{Y}$ feels sorry for $\mathrm{X}$. If so, then on the face of it, $\mathrm{X}$ and $\mathrm{Y}$ experience the same emotion: they both feel sorry. Should that count as a clear-cut case of empathy (Deonna 2007; Zahavi 2011; Michael 2014)? Not necessarily. Both feel sorry, but the intentional object of their respective sorrow is entirely different. One feels sorry about her husband's disease, and the other feels sorry about her friend's state of mind. ${ }^{1}$

1. In fact, there is some room for stipulation here. One can stress the difference between the intentional object of X's and Y's respective feeling (Zahavi 2011). But one can also 
Now Michael $(2014,160,169)$ asks to what extent the interpersonal similarity condition should apply to the intentional object of emotional empathy. It is controversial whether physical pain has an intentional object at all but uncontroversial that fear and disgust do. We just argued that although $\mathrm{X}$ and $\mathrm{Y}$ both feel sorry, $\mathrm{Y}$ nonetheless fails to empathize with $\mathrm{X}$ in virtue of the difference between the intentional content of respectively $X$ 's and Y's feeling. Instead, it is a mere coincidence if Y's feeling sorry about X overlaps with X's feeling sorry about her husband's medical condition. Y's feeling sorry is better construed as an example of sympathy for $\mathrm{X}$ rather than empathizing with X. Our nuanced response to Michael is thus that the intentional object of one individual's emotion stands to the intentional object of another's empathetic response in the determinate/determinable relation, whose limit is identity. It is compatible with the interpersonal condition on empathy that the intentional object of a vicarious emotion is generally less determinate than that of the standard emotion that caused it. For instance, $\mathrm{X}$ is afraid of a specific bully at school, whereas $\mathrm{Y}$, who empathizes with $\mathrm{X}$, is vicariously afraid of bullies whoever they are. This suggests that acceptance of the interpersonal similarity condition on empathy does not always require detailed background knowledge about the person one empathizes with.

Hence, the interpersonal similarity condition on empathy enables us to distinguish empathy from both nonempathetic mind-reading and sympathy. However, this does not entail that it is sufficient, as the following example shows. Suppose that individuals $\mathrm{X}$ and $\mathrm{Y}$ are both afraid as a result of hearing a dog's loud barking. In this case, $X$ and $Y$ share their standard fear as a result of a common cause (the dog's barking). But neither needs empathize with the other. In fact, neither experiences vicarious fear. But empathetic fear is vicarious fear, that is, fear that resembles another's standard fear that caused it.

We have argued that the involvement of affective sharing in empathy is supported by recent findings in social neuroscience showing overlap of brain activity between one individual experiencing some specific affective state (e.g., pain, disgust, fear) and the same individual being provided with cues that another individual is in the very same affective state (Wicker et al. 2003; Keysers et al. 2004; Singer et al. 2004; Vignemont and Jacob 2012). However, as we will now see, our interpretation of the neuroscientific findings has been challenged by Michael (2014) and Michael and Fardo (2014).

3. The Pain Matrix Reloaded. In 2012, we focused on pain and we took as our starting point the widely recognized dual nature of painful subjective

stress the similarity between X's and Y's feeling on the grounds that the intentional object of the former is part of the intentional object of the latter (Jacob 2011). 
experiences: physical (or standard) pain has both a sensory-discriminative component (intensity of pain and its bodily location) and an affective component (unpleasantness of pain). We have argued that an experience of $v i$ carious pain can be primarily - but by no means exclusively — generated by the selective activation of either the sensory-discriminative or the affective component. For instance, using one experimental paradigm, Avenanti et al. (2005) found that seeing a needle deeply penetrate another's hand causes in the observer the same sensorimotor response (i.e., muscle-specific freeze) as in the person whose hand is being penetrated. By contrast, using a different experimental paradigm, Singer et al. (2004) found that experiencing pain and being aware of another's pain selectively activate the same affective component of the pain neural matrix with no activation of the sensorimotor component. When participants were explicitly asked to pay more attention to the intensity of pain or to its bodily location, both the affective and the sensory components of pain were activated (e.g., secondary somatosensory cortex; cf. Cheng et al. 2007; Lamm, Batson, and Decety 2007). However, there was no somatotopic organization of the brain responses (and no activity in the primary somatosensory cortex). In other words, vicarious affective pain was not encoded in a particular body part. Thus, it seems as if there are two types of vicarious pain, one of which is selectively correlated with a specific body part, whereas the bodily location of pain is irrelevant to the other one. Furthermore, the former is automatic (Avenanti et al. 2006), but the latter can be inhibited and is subject to top-down modulation by a wide range of factors (for a review, see Engen and Singer 2013).

However, Michael (2014) and Michael and Fardo (2014) question the claim that contagious responses to another's emotional or affective experience primarily involve the activation of the sensory-discriminative component of one's own emotional or affective system, at the expense of the affective component. As we argue below, there is evidence that the sensorydiscriminative component of one's pain system constitutes vicarious contagious pain (Avenanti et al. 2006; Garbarini et al. 2015). Still the dissociation between the sensory and the affective components of pain in vicarious experiences is only partial. But 'pure' dissociations are rarely, if ever, found in cognitive neuroscience. We defer to section 5 our response to the question whether we can extend our account of vicarious pain to vicarious emotions.

Second, Michael (2014) and Michael and Fardo (2014) argue that recent work by Iannetti and colleagues showing that activation of the pain matrix is not restricted to responses to nociceptive stimuli casts doubt on our account of empathetic pain. We disagree. On the one hand, Iannetti et al. (2013) have argued that overlap of brain activity between physical pain and social pain (caused by social exclusion) cannot show that social pain "hurts." On the other hand, Legrain et al. (2011) report that the pain matrix 
can be activated in response not merely to nociceptive stimuli but also to salient visual, auditory, or tactile stimuli in the space immediately surrounding the body. If so, then arguably the pain matrix should be relabeled the alarm matrix, which can be activated by all sorts of threats lying close to the body or on the body. For example, awareness that another person is in pain can also trigger the alarm.

Arguably these findings shed light on the nature of physical pain itself: pain is an alarm system. If so, then the affective component of pain is the evaluative component of this alarm system: by offering a negative evaluation of an actual or potential threat to one's bodily integrity, it motivates an appropriate response (Cutter and Tye 2011; Bain 2013). ${ }^{2}$ The affective component is associated with the dedicated sensory component of pain when the disturbance falls within the limits of the body. If the disturbance lies immediately outside the body and may harm it, then the affective component can also be associated with other sensory representations - visual or auditory (Vignemont, forthcoming).

On this account, empathetic pain (i.e., vicarious affective pain) is generated by the evaluative activity of the affective component of one's pain system because the affective component of the pain system works mostly as an alarm system that evaluates and motivates responses to threats. In standard pain, the affective component of one's pain system is triggered by the detection of threats to one's own body. But it can also be activated by the detection of stimuli that are threats not to one's own body but to another's body instead. Empathetic pain is thus isomorphic to physical pain to some extent. Far from disproving our account of empathetic pain, the findings by Iannetti and colleagues showing that an individual's pain matrix can be activated in the absence of nociceptive stimuli are consistent with our account.

4. The Ascription Condition. As we read it, the neuroscientific evidence shows not only that the brain activity underlying vicarious pain partially overlaps with the brain activity underlying physical pain but also that there are two kinds of vicarious experiences of pain. Unlike vicarious sensory pain, vicarious affective pain is not localized in a particular bodily part. We interpret this dissociation in terms of the conceptual distinction between contagious and empathetic responses. On our view, what matters to the distinction between contagious and empathetic responses to another's pain is the ascription condition.

2. As Bain $(2013,82)$ puts it, "a subject's being in unpleasant pain consists in his (i) undergoing an experience (the pain) that represents a disturbance of a certain sort, and (ii) that same experience additionally representing the disturbance as bad for him in the bodily sense." 
Let us first consider sensory vicarious pain. As we mentioned earlier, Avenanti et al. (2005) reported that seeing another's hand being subjected to painful stimulation causes motor inhibition in participants' corresponding own hand. Interestingly, this response seems to be primarily self-centered, as shown by the following findings. First, the effect was not increased when participants explicitly adopted the target's perspective. In a follow-up study, Avenanti et al. (2006) found indeed no difference when participants were asked respectively to focus on the qualities of the painful event or to mentally simulate the target's pain. One would have expected the opposite result if the motor response was other-directed. Second, they did not find any correlation between the strength of the response and the participants' score on empathy questionnaires. Finally, a recent study using the same experimental paradigm recorded motor inhibition only when the hand that the needle penetrated was presented from a first-person visuospatial perspective but not when it was presented from a third-person perspective (Garbarini et al. 2015). Following Avenanti and colleagues (2009) and Garbarini and colleagues (2015), we thus propose to interpret vicarious sensory pain in terms of self-centered contagious pain. When seeing another's hand subjected to painful stimulation, while knowing nothing about whose hand it is, one maps the other's bodily part subjected to painful stimulation onto one's own bodily counterpart, and one anticipates the sensorimotor consequences of pain at this bodily location. As a result, one's experience of vicarious pain is both anticipatory and entirely self-centered: it is an instance of contagious pain, not empathetic pain. By contrast, one vicariously experiences the unpleasantness of another's pain by activating the affective component of one's own pain system. This does not require pain to be represented at a definite bodily location. Unlike vicarious sensory pain, vicarious affective pain is other-directed (e.g., Singer et al. 2006). The most conclusive corroboration comes from the following study. Participants were told that some patients reacted with pain when they received a soft touch but not when they were pinpricked. It was found that participants displayed activity in the affective component of pain only when they saw the patients being touched by a Q-tip (Lamm, Meltzoff, and Decety 2010). Following these findings, we propose to interpret affective vicarious pain in terms of other-directed empathetic pain.

In a nutshell, contagious pain and empathetic pain are two distinct vicarious experiences of pain. Whereas the former is self-centered, the latter is other-directed. We suggest that the direction of intentionality (i.e., selfcentered vs. other-directed) is determined by whether it is primarily the sensory or the affective component of pain that is vicariously activated. These differences between the two types of vicarious experiences help us understand why affective vicarious experiences alone can meet the ascription condition. In either standard pain or contagious pain, the unpleasant- 
ness of actual or hypothetical pain is correlated with the localization of pain in some definite bodily part. By contrast, in vicarious affective pain, there is an asymmetry between the strong activity of the affective component (which generates a strong psychological disarray) and the weak activity of the sensory component of the pain system (which generates a weak global bodily feeling). The lack of bodily location makes empathetic pain a highly specific type of pain. One can mislocalize standard pain (e.g., referred pain), but one can never experience standard pain without ascribing it to a rough bodily location.

The experience of the unpleasantness of standard pain motivates a selective range of bodily movements, whose function is to prevent or alleviate actual or potential pain (e.g., remove your hand from the hot stove), which is driven by the bodily location of pain conveyed by the sensory component of pain. However, the feeling of empathetic pain has no definite bodily location, to the extent that the sensory component of pain is not active at all or very weakly so. Consequently, no definite sensorimotor expectations can be generated. Lacking definite sensorimotor expectations about the consequences of pain at a definite bodily location, one feels instead a global bodily feeling of the unpleasantness of generic pain. As a result, one becomes aware that one's own psychological disarray is being caused by another's standard pain. This, we surmise, is why experiences of empathetic pain alone meet the ascription condition.

This process, however, may seem overly complex. For instance, Michael and Fardo (2014) offer what looks like the simpler suggestion that vicarious experiences of pain are bound to be other-directed from the very start, in virtue of their perceptual origins. They assume that it is sufficient for meeting the ascription condition that a vicarious experience of pain results from the perception of other people in pain. Their suggestion, however, fails to account for the difference between contagious and empathetic vicarious states. If they agree that only the latter, not the former, can contribute to affective mind-reading, as they seem willing to, then the reason must lie not in what they have in common but instead in what makes them different from one another. All vicarious experiences of pain share the same kinds of inputs: awareness of cues indicating another's standard pain. So the distinctive otherdirectedness of empathetic vicarious pain cannot directly stem from the inputs to both kinds of vicarious experiences of pain. It must be generated at a later stage in the process whereby one becomes primarily aware of the activity of the affective component of one's own pain system, at the expense of the sensorimotor component.

5. Beyond Empathy for Pain. The last challenge for our account is its limited scope, which seems restricted to empathetic pain. Pain, however, is far from being a prototypical emotion. The crucial question is whether it 
makes sense to draw a distinction between two kinds of vicarious responses in the case of other emotions (e.g., fear and disgust). Does it make sense to distinguish contagious fear (or disgust) from empathetic fear (or disgust), where the former is supposed to be fundamentally self-centered and the latter fundamentally other-directed? In other words, the question is, what is it about the content of contagious fear (or contagious disgust) that makes it self-centered? What is it about the content of empathetic fear (or disgust) that makes it other-directed?

All emotions may not have the same dual nature as pain. Still, on some accounts at least, they can be characterized in terms of two distinct dimensions, namely, their evaluative and their bodily dimensions. Most conceptions of the emotions have either overintellectualized them or overembodied them. On the one hand, some approaches have focused on the intentionality of the emotions (e.g., fear or disgust of something), thereby accounting for emotions in purely cognitive terms (Solomon 1993). On the other hand, other approaches have focused on the phenomenology of the emotions (e.g., I feel frightened), thereby accounting for some (if not all) emotions in terms of experiencing bodily changes (e.g., James 1884; Damasio 1999; Prinz 2004). Some recent proposals, however, suggest an intermediate approach, according to which emotions are both bodily and evaluative attitudes: "we understand why emotions are evaluations once we admit that they relate to values by virtue of being experiences of one's body being ready or poised to act in some specific manner towards a given object or situation" (Teroni and Deonna 2014, 28). Emotions have two fundamental dimensions: on the one hand, as their phenomenology shows, they are anchored to basic bodily feelings. On the other hand, they have a basic evaluative function: to experience an emotion is to evaluate or appraise some event, fact, property, or object in a distinctive way, which is in turn revealed by some specific associated action readiness. ${ }^{3}$ We argue that each of the two basic components of standard emotions can be mapped onto each of the two kinds of vicarious emotions.

Let us first consider contagious experiences. For example, I am in the middle of a crowd and someone starts panicking. The panic automatically spreads to everybody, including me. What do I experience? It seems relatively uncontroversial that I experience contagious fear. I feel afraid, but whether I feel afraid of something definite or not, I do feel my heart beating faster and also the urge to run as much as everybody else around. My contagious fear is primarily driven by the bodily feelings associated with fear, not by the evaluative affective component of fear. If I am aware of

3. The evaluative attitude can be about an external nonbodily object or event (e.g., a lion), but it can also be about the subject's own body (i.e., reflexive emotions). Even in this latter case, the distinction between the two dimensions holds: the body is both a source of feelings and an intentional object. 
the immediate source of my vicarious fear, this information is conveyed by other cues. In a nutshell, my vicarious fear is strongly embodied. This is why most instances of emotional contagion are described in embodied rather than in affective terms: one talks of contagious crying or contagious laughter rather than contagious distress or contagious happiness. Similarly, experiences of vicarious sensory pain are vicarious experiences of strongly embodied aspects of pain: they are primarily self-centered and represent distinctive bodily parts.

By contrast, suppose I perceive cues of a child's fear of a lion behind bars in a zoo. I may not be afraid of the lion myself. Nonetheless, even if I am not, I can still vicariously feel the child's fear of the lion. Clearly, my vicarious fear of the lion is quite different from my undirected contagious fear caused by crowd panic. What primarily drives empathetic fear is the affective evaluative component of fear, not the bodily feelings associated with fear. My contagious fear need not represent any intentional object. My empathetic fear, however, must be directed and be about something (e.g., the lion). More specifically, my vicarious fear of the lion consists in an evaluative representation of the lion as dangerous.

But how does my empathetic fear of the lion differ from the child's standard fear? How can it meet the ascription condition? We assume that an agent's standard emotion involves both an evaluative appraisal and a bodily feeling, both anchored to the agent's own bodily perspective. Now the evaluative component of an agent's standard emotional experience involves a distinctive set of parameters. On the one hand, danger is always appraised relative to some agent: what is dangerous for a young child is not necessarily dangerous for a healthy adult. On the other hand, the evaluative component of an agent's fear involves standards of appraisal of the danger of a threatening stimulus, relative to the agent's own cognitive resources and values. For example, the evaluative component of the child's experience of fear involves an appraisal of the danger of the lion behind bars in the zoo, at a location near the child's body, relative to the child's own values and cognitive resources.

An experience of either standard fear or contagious fear is primarily selfcentered: it is likely to directly cause one to run away from the source of the fearful experience in order to protect oneself. But what underlies the experience of empathetic vicarious fear is primarily the activity of the evaluative component of one's own fear system (at the expense of the bodily feeling of fear). In empathetic vicarious fear, there may be a discrepancy between danger as appraised by one's own standards and one's awareness of the cues of another's fear. If so, given that by one's own standards of appraisal of danger, one should not experience fear at all, one must shift one's own standards in order to make sense of the cues of another's fear. In the case of pain, empathetic pain is generated by running offline the affec- 
tive component of one's pain system (Vignemont and Jacob 2012). In the case of empathetic fear, one appraises danger according to someone else's standards of evaluation by running offline one's fear system. This is what it takes to respond empathetically to another's cues of fear: one uses standards of appraisal of danger that belong to someone else so that one can run offline the evaluative component of one's fear system. If one does not share those standards, then one must shift one's own standards in order to match the other's standards. Consequently, experiences of empathetic fear whereby one runs offline the evaluative component of one's fear system are fundamentally other-directed. Hence, what makes a vicarious experience otherdirected, and thus empathetic, are (i) the fact that it necessarily consists of an evaluative attitude and (ii) the fact that the evaluation is performed on the basis of another individual's standards. For example, I am able to appraise the presence of the lion behind bars as dangerous for myself and the child, according to the child's cognitive resources and values. Thus, if and when I experience empathetic vicarious fear, I am not tempted to run away from the lion at all but instead to move toward the child and to comfort her by trying to change her standards of appraisal of danger by, for example, pointing to the protective bars. ${ }^{4}$

To conclude, we made four basic points: to empathize with another's emotion is not the same as nonempathetically mind-reading her emotion. Empathetic emotional experiences further differ from sympathetic responses to others' emotions because they do not have the same evaluative content. Third, they differ from contagious experiences because they are evaluative attitudes. Finally, they differ from standard emotions because in empathetic emotional experiences, one may shift one's standards of evaluation relevant to a given emotion to match another's standards of evaluation. In virtue of these specificities, empathetic experiences meet both the interpersonal similarity and the ascription condition. So our account of empathy in terms of interpersonal similarity allows us to distinguish it from other-related social attitudes not only in the case of pain but also in the case of other emotions.

\section{REFERENCES}

Avenanti, Alessio, D. Bueti, G. Galati, and S. Aglioti. 2005. "Transcranial Magnetic Stimulation Highlights the Sensorimotor Side of Empathy for Pain." Nature Neuroscience 87:955-60. Avenanti, Alessio, I. Minio-Paluello, I. Bufalari, and S. Aglioti. 2006. "Stimulus-Driven Modulation of Motor-Evoked Potentials during Observation of Others Pain." Neuroimage 321:31624

4. The standard experience of disgust also involves an evaluative component. One can also experience vicarious empathetic disgust by running offline the evaluative component of one's disgust system in order to match another's standards of appraisal of dangerous food. 
Avenanti, Alessio, I. Minio-Paluello, A. Sforza, and S. Aglioti. 2009. "Freezing or Escaping? Opposite Modulations of Empathic Reactivity to the Pain of Others." Cortex 45 (9): 1072-77. Bain, David. 2013. "What Makes Pains Unpleasant?” Philosophical Studies 166 (1): 69-89.

Cheng, Yawei, C. P. Lin, H. L. Liu, Y. H. Hsu, K. E. Lim, D. Hung, and J. Decety. 2007. "Expertise Modulates the Perception of Pain in Others." Current Biology 17 (19): 1708-13.

Cutter, Brian, and Michael Tye. 2011. "Tracking Representationalism and the Painfulness of Pain." Philosophical Issues 21 (1): 90-109.

Damasio, Antonio. 1999. The Feeling of What Happens. London: Heinemann.

Deonna, Julien. 2007. "The Structure of Empathy." Journal of Moral Philosophy 4 (1): 99-116.

Engen, Hakon, and Tania Singer. 2013. "Empathy Circuits." Current Opinion in Neurobiology 23 (2): 275-82.

Garbarini, Francesca, G. Bucchioni, C. Fossatar, V. Bruno, A. Cavallo, L. Pia, and M. NeppiModona. 2015. "Empathy or Ownership? Evidence of Corticopinal Modulation during Pain Observation." Paper presented at the 5th Conference of the Federation of the European Societies of Neuropsychology, Tampere, Finland, September 9-11.

Iannetti, Giandomenico, T. V. Salomons, M. Moayedi, A. Mouraux, and K. D. Davis. 2013. "Beyond Metaphor: Contrasting Mechanisms of Social and Physical Pain." Trends in Cognitive Sciences 17 (8): 371-78.

Jacob, Pierre. 2011. "The Direct-Perception Model of Empathy: A Critique." Review of Philosophy and Psychology 2 (3): 519-40.

James, William. 1884. "What Is an Emotion?" Mind 9:188-205.

Keysers, Chistian, B. Wicker, V. Gazzola, J. L. Anton, L. Fogassi, and V. Gallese. 2004. "A Touching Sight: SII/PV Activation during the Observation and Experience of Touch." Neuron 42:335-46.

Lamm, Claus, D. Batson, and J. Decety. 2007. "The Neural Substrate of Human Empathy: Effects of Perspective-Taking and Cognitive Appraisal." Journal of Cognitive Neuroscience 191:42-58

Lamm, Claus, A. Meltzoff, and J. Decety. 2010. "How Do We Empathize with Someone Who Is Not like Us? A Functional Magnetic Resonance Imaging Study." Journal of Cognitive Neuroscience 22:362-76.

Legrain, Valery, G. D. Iannetti, L. Plaghki, and A. Mouraux. 2011. "The Pain Matrix Reloaded: A Salience Detection System for the Body." Progress in Neurobiology 93 (1): 111-24.

Michael, John. 2014. "Towards a Consensus about the Role of Empathy in Interpersonal Understanding." Topoi 33 (1): 157-72.

Michael, John, and Francesca Fardo. 2014. "What (If Anything) Is Shared in Pain Empathy? A Critical Discussion of de Vignemont and Jacob's Theory of the Neural Substrate of Pain Empathy." Philosophy of Science 81 (1): 154-60.

Prinz, Jesse. 2004. Gut Reactions: A Perceptual Theory of Emotion. New York: Oxford University Press.

Scheler, Max. 1954. The Nature of Sympathy. Trans. P. Heath. London: Routledge \& Kegan Paul.

Singer, Tania, B. Seymour, J. O’Doherty, H. Kaube, R. Dolan, and C. D. Frith. 2004. "Empathy for Pain Involves the Affective but Not Sensory Components of Pain.” Science 303:1157-62.

Singer, Tania, B. Seymour, J. O’Doherty, K. Stephan, R. Dolan, and C. D. Frith. 2006. "Empathic Neural Responses Are Modulated by the Perceived Fairness of Others." Nature 439:466-69.

Solomon, Robert C. 1993. "The Philosophy of Emotions." In Handbook of Emotions, 3rd ed., ed. Michael Lewis, Jeannette M. Haviland-Jones, and Lisa Feldman Barrett, 3-15. New York: Guilford.

Teroni, Fabrice, and Julien Deonna. 2014. "In What Sense Are Emotions Evaluations?" In Emotion and Value, ed. Sabine Roeser and Cain Todd. Oxford: Oxford University Press.

Vignemont, Frédérique, de. Forthcoming. "Can I See Your Pain? An Evaluativist Model of Pain Perception." In Routledge Handbook of Philosophy of Pain, ed. Jennifer Corns. London: Routledge.

Vignemont, Frédérique, de, and Pierre Jacob. 2012. "What Is It like to Feel Another's Pain?" Philosophy of Science 79:295-316.

Vignemont, Frédérique, de, and Tania Singer. 2006. "The Empathic Brain: How, When and Why?" Trends Cogn Sci 10 (10): 435-41. 
Wicker, Bruno, C. Keysers, J. Plailly, J. P. Royet, V. Gallese, and G. Rizzolatti. 2003. "Both of Us Disgusted in My Insula: The Common Neural Basis of Seeing and Feeling Disgust." Neuron 40:655-64.

Zahavi, Dan. 2011. "Empathy and Direct Social Perception: A Phenomenological Proposal." Review of Philosophy and Psychology 2 (3): 541-58. 